\title{
Access to credible information on schizophrenia patients' medication adherence by prescribers can change their treatment strategies: evidence from an online survey of providers
}

\author{
This article was published in the following Dove Press journal: \\ Patient Preference and Adherence \\ 27 June 2017 \\ Number of times this article has been viewed
}

Jason Shafrin,' Suepattra G May, 'Anshu Shrestha,' Charles Ruetsch, ${ }^{2}$ Nicole Gerlanc, ${ }^{2}$ Felicia Forma, ${ }^{3}$ Ainslie Hatch, ${ }^{4}$ Darius N Lakdawalla, ${ }^{1,5}$ Jean-Pierre Lindenmayer ${ }^{6}$

'Precision Health Economics, Los Angeles, CA, ${ }^{2}$ Health Analytics, Columbia, MD, ${ }^{3}$ Otsuka Pharmaceutical Development \& Commercialization, Inc., ${ }^{4} \mathrm{ODH}$, Inc., Princeton, NJ, ${ }^{5}$ Schaeffer Center for Health Policy and Economics, University of Southern California, Los Angeles, CA, ${ }^{6}$ Department of Psychiatry, New York University School of Medicine, New York, NY, USA

Video abstract

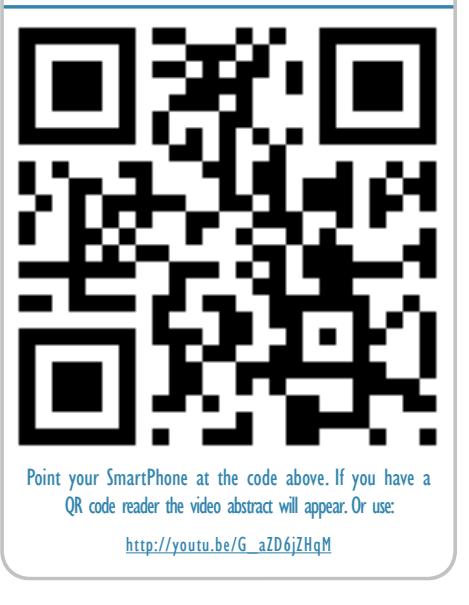

Correspondence: Jason Shafrin Precision Health Economics, II 100 Santa Monica Blvd, Suite 500, Los Angeles, CA 90025, USA

Tel + I 3109847705

$\mathrm{Fax}+13109826311$

Email jason.shafrin@precisionhealtheconomics. com
Objective: Overestimating patients' medication adherence diminishes the ability of psychiatric care providers to prescribe the most effective treatment and to identify the root causes of treatment resistance in schizophrenia. This study was conducted to determine how credible patient drug adherence information (PDAI) might change prescribers' treatment decisions.

Methods: In an online survey containing 8 clinical case vignettes describing patients with schizophrenia, health care practitioners who prescribe antipsychotics to patients with schizophrenia were instructed to choose a preferred treatment recommendation from a set of predefined pharmacologic and non-pharmacologic options. The prescribers were randomly assigned to an experimental or a control group, with only the experimental group receiving PDAI. The primary outcome was the prescribers' treatment choice for each case. Between-group differences were analyzed using multinomial logistic regression.

Results: A convenience sample ( $\mathrm{n}=219$ ) of prescribers completed the survey. For 3 nonadherent patient vignettes, respondents in the experimental group were more likely to choose a longacting injectable antipsychotic compared with those in the control group $(77.7 \%$ experimental vs $25.8 \%$ control; $P<0.001)$. For 2 adherent but poorly controlled patient vignettes, prescribers who received PDAI were more likely to increase the antipsychotic dose compared with the control group $(49.1 \%$ vs $39.1 \% ; P<0.001)$. For the adherent and well-controlled patient vignette, respondents in both groups made similar treatment recommendations across all choices $(P=0.099)$, but respondents in the experimental arm were more likely to recommend monitoring clinical stability (87.2\% experimental vs $75.5 \%$ control, reference group).

Conclusion: The results illustrate how credible PDAI can facilitate more appropriate clinical decisions for patients with schizophrenia.

Keywords: adherence, case vignettes, long-acting injectables, schizophrenia, treatment decision

\section{Introduction}

Adherence to an antipsychotic medication regimen helps to prevent relapse and hospitalization in patients with schizophrenia. ${ }^{1-3}$ By contrast, poor adherence impedes recovery, ${ }^{4,5}$ negatively affects the functioning and quality of life of patients, ${ }^{3,6}$ and is associated with higher health care costs. ${ }^{1,7}$ Despite its importance in the management of ongoing psychiatric care, monitoring patient compliance with medication remains a challenge because the reliability and validity of most of the measures of medication adherence are questionable. A source of real-time, credible (ie, accurate 
and reproducible), case-level data on medication adherence that is readily accessible to psychiatric prescribers to guide treatment decisions is needed. ${ }^{8}$

Available methods of assessing patient-level adherence can be divided into subjective and objective measures. Subjective measures include patient self-reports or interviewer ratings, which have questionable reliability. ${ }^{9,10}$ Clinicians and care staff often overestimate adherence using such measures. ${ }^{11}$ Pharmacy records, electronic monitoring systems, such as the MEMSCap ${ }^{\mathrm{TM}}$ Medication Event Monitoring System (WestRock Healthcare, Norcross, GA, USA), and monitoring for serum levels of antipsychotic drugs do not directly record medication ingestion in real time. ${ }^{8}$ Alternatively, newer technologies, such as video-observed therapy ${ }^{12}$ and a digital medicine system using medication embedded with an ingestible sensor, ${ }^{13,14}$ allow for direct observation and recording of an ingestion event, respectively, thereby providing credible estimates of adherence. Such information has the potential to lead to better-informed clinical decisions. However, there is limited evidence on whether prescribers would make use of or attend to such information if it were available.

The objective of this study was to determine how access to credible patient drug adherence information (PDAI) might influence psychiatric practitioners' prescribing and practice patterns. There has been considerable research on the issue of adherence in patients with schizophrenia, and specifically on the real-world challenges that providers encounter while attempting to gauge whether patients are adherent to their medication. ${ }^{15}$ Other studies have used survey vignettes to examine how patient and provider characteristics impact antipsychotic treatment strategies. ${ }^{16,17}$ Nevertheless, to our knowledge, this study is the first to use a 2-arm randomized controlled online survey design to test whether better information about adherence leads to more appropriate treatment decision-making among providers treating patients with serious mental illnesses.

\section{Methods Study design}

A convenience sample of health care practitioners who treat schizophrenia with antipsychotics, assigned to experimental or control groups using simple randomization, participated in this cross-sectional survey. The survey contained clinical case vignettes that described patients with schizophrenia being treated with antipsychotic medication. The 2 groups received identical vignettes with one exception: the experimental group's vignettes included credible PDAI, whereas the control group's vignettes did not. As a test of similarity across the experimental and control respondents, both groups also received 2 vignettes that were completely identical in all respects. Following each vignette, respondents were asked what, if any, changes they would make to the current treatment regimen by selecting from lists of both pharmacologic and non-pharmacologic treatment options. Information on respondents' demographics, training, clinical practice setting, and approaches to treatment was also collected. The Chesapeake Institutional Review Board reviewed the study procedures and exempted the study from full review because of low/no risk to the study participants.

\section{Study participants}

A convenience sample of psychiatric prescribers, including psychiatrists, nurse practitioners, and physician assistants, currently treating patients with schizophrenia was invited by telephone, email, mail, and/or fax to participate in the study. We elected to include both psychiatrists and nonpsychiatrist prescribers as the latter account for a meaningful fraction of atypical antipsychotic prescriptions. ${ }^{18}$ Eligible participants included prescribers who had graduated from an accredited medical school, prescribed antipsychotics, and treated $\geq 1$ patient with schizophrenia per month. The study included doctors of medicine, doctors of osteopathic medicine, physician assistants, and nurse practitioners. Those who did not meet the eligibility criteria or refused to complete the eligibility screener were excluded, whereas all respondents who successfully completed the survey, including pilot test participants, were compensated for their time and effort. Participants were blinded to the sponsor of the study.

The study was powered such that a target sample size of $n=200$ participants assigned to 2 equal groups would be sufficient to detect a difference of $16.5 \%$ in the proportion of participants opting for adherence-focused treatment in the presence of adherence information at 0.05 alpha level and with a power of 0.80 .

\section{Survey}

The survey incorporated 8 clinical case vignettes, which were brief, carefully written descriptions of patients with schizophrenia in situations that simulate real-world clinical scenarios. The vignettes were drawn originally from case vignettes in peer-reviewed journals, but then modified using input from a clinical expert (Lindenmayer) to simplify each vignette and modify it for the purposes of this study. Key patient characteristics (age, sex, disease severity, and adherence) varied across the 8 vignettes (Table S1). Each vignette 
represented specific patient behaviors and characteristics, including self-reported medication adherence, substance use, and the presence of medication side effects, as well as both self-reported and observed symptom severity. Based on specified combinations of these patient characteristics and behaviors, each vignette was assigned to one of the following types: (1) nonadherent patients; (2) adherent, poorly controlled patients; and (3) adherent, well-controlled patients. All vignettes represented patients using oral antipsychotic medication at the time of presentation. A sample vignette is provided in Figure 1, and the full text of all vignettes is provided in Table $\mathrm{S} 2$.

In addition to the vignettes, the survey included questions of provider and practice characteristics including years of practice, current number of schizophrenic patients under their care, the number currently on antipsychotic medication, and the methods used to assess patient compliance with medication. The survey was programmed to be accessible online and adaptable to both desktop and mobile devices using Qualtrics survey software (Qualtrics, Provo, UT, USA).

\section{Survey administration}

Study participants received an e-mail with a link to the online survey site with credentials. After completing an informed consent form, participants were provided with a set of instructions, and then asked a series of questions to determine whether they met the study inclusion criteria. Those who met the inclusion criteria were presented with 8 clinical vignettes. As previously mentioned, the vignettes presented to the experimental group included credible PDAI (eg, information from MEMSCap, blood levels), whereas the control group received the same vignettes with no credible PDAI. To test for differences in treatment patterns between the control and experimental groups not associated with the experimental condition,

Robert is a 44-year-old male with a 17 -year history of schizophrenia who has had multiple psychiatric admissions usually in the context of non-adherence. Admissions have been characterized by gradually increasing isolation, preoccupations and insomnia. These symptoms escalate eventually to agitation, intense paranoid ideation with at times yelling at strangers and total neglect of his ADLs. Robert has been living in a group home with 8 other residents where he is responsible for taking his own medication. He usually does attend his monthly outpatient visits and also attends a club house. His medications were lurasidone (Latuda) $(80 \mathrm{mg} / \mathrm{d})$ and metformin $(1,000 \mathrm{mg} / \mathrm{d})$. Over the past few weeks Robert has become increasingly unpredictable, responding to internal stimuli with bursts of uncontrollable laughing and swearing. He also believes the other group home members are out to get him. His psychiatrist called 911 to have Robert admitted to the inpatient services. After a 6-day intensive inpatient stay, Robert re-stabilized on lurasidone (Latuda) (120 mg/d) and was discharged back to the group home. [Two weeks after discharge, his psychiatrist reviewed Robert's electronic cap monitor and blood plasma levels and found that Robert had only taken half of the pills he was supposed to take and had low plasma levels of lurasidone].

Information in italics was provided to the experimental group, not the control group.

\section{Pharmacologic Treatment Options}

Please select the most likely course of action you would take for this patient. Select one:

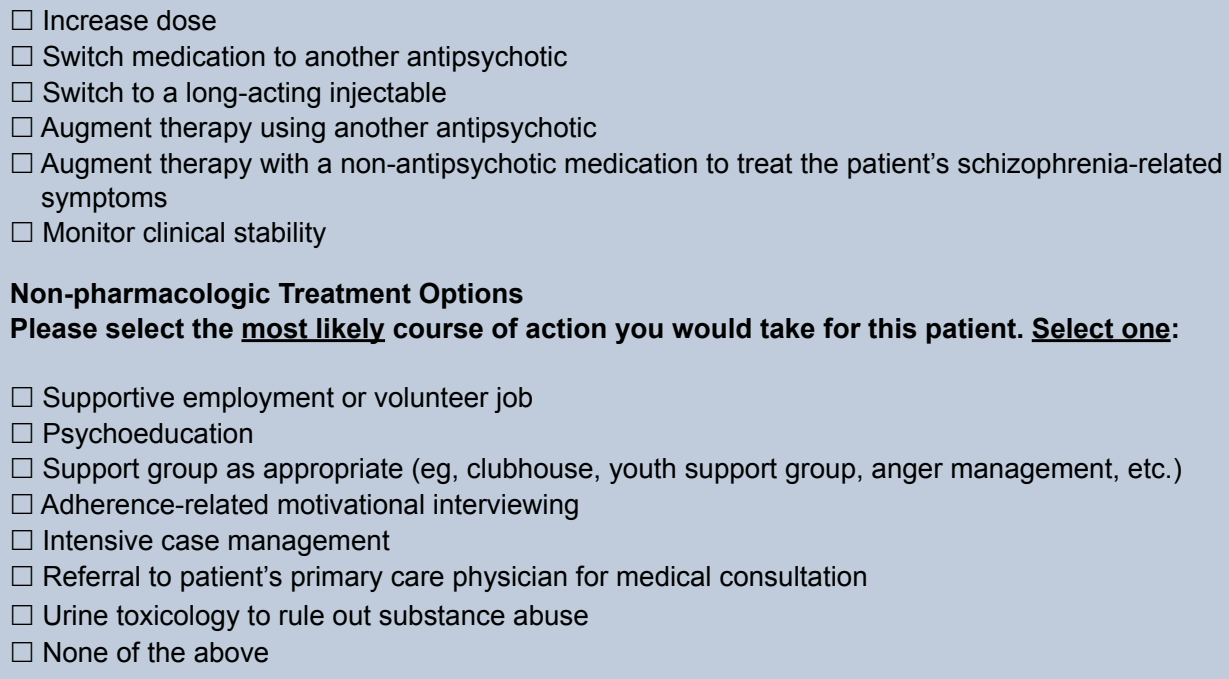

Figure I Example of a case vignette and survey treatment options. 
respondents in both groups received 2 vignettes (1 with and 1 without PDAI) that were identical in all respects.

In response to each vignette, participants were asked to select their preferred pharmacologic (increase dose, switch to another oral antipsychotic medication, switch to a long-acting injectable [LAI] medication, augment therapy using another antipsychotic, augment therapy with a non-antipsychotic medication to treat symptoms, or monitor clinical stability) and non-pharmacologic treatment recommendations (supportive employment, psychoeducation, support group, adherence-related motivational interviewing, intensive case management, referral to patient's primary care physician, or ruling out substance abuse).

Following the 8 survey vignette questions, additional information on the respondent's demographics, patient population, and treatment practices was requested. Finally, the survey presented a statement of appreciation for participating in the study and requested each respondent's mailing address in order to mail the remuneration for completing the survey.

\section{Analysis}

The primary end point for this study was the prescribers' selection of treatment modification based on the list of pharmaceutical options. All variables of interest were categorical, with frequency distributions and percentages calculated for the total sample as well as by type of cases mentioned above. A Wald chi-squared $\left(\chi^{2}\right)$ test of homogeneity was performed on frequencies of provider and practice characteristics to assess if any of the variables were significantly different between the experimental and control groups. The $\chi^{2}$ test was also used to determine between-group differences in proportions of respondents who selected each of the treatment choices. Pairwise comparisons by treatment choice across groups were performed using multinomial logistic regression, with false discovery rate adjustment applied to the resulting $P$-values. The responses of "monitoring clinical stability" and "intensive case management" served as the reference groups for responses to pharmacologic and non-pharmacologic treatment, respectively.

\section{Results}

\section{Survey respondents}

Of the 240 prescribers who responded to the survey invitation, $224(93.3 \%)$ were randomized and 219 of them $(97.8 \%)$ completed the survey (Figure 2). Among the respondents, $127(58.0 \%)$ were female, $133(60.7 \%)$ were white, and most $(62.1 \%)$ had practiced medicine for $>5$ years. Provider demographic and practice characteristics were well balanced across groups (Table 1). The majority of respondents indicated that they assess patients' adherence in their practice by asking an informant such as a relative or a caregiver $(n=205 ; 93.6 \%)$, explicitly asking the patient $(n=203 ; 92.7 \%)$, or by assessing patient symptomatology $(n=175 ; 79.9 \%)$.

\section{Clinical case vignettes}

\section{Pharmacologic responses}

Validation cases

To identify any between-group differences in providers' treatment preferences not associated with the experimental

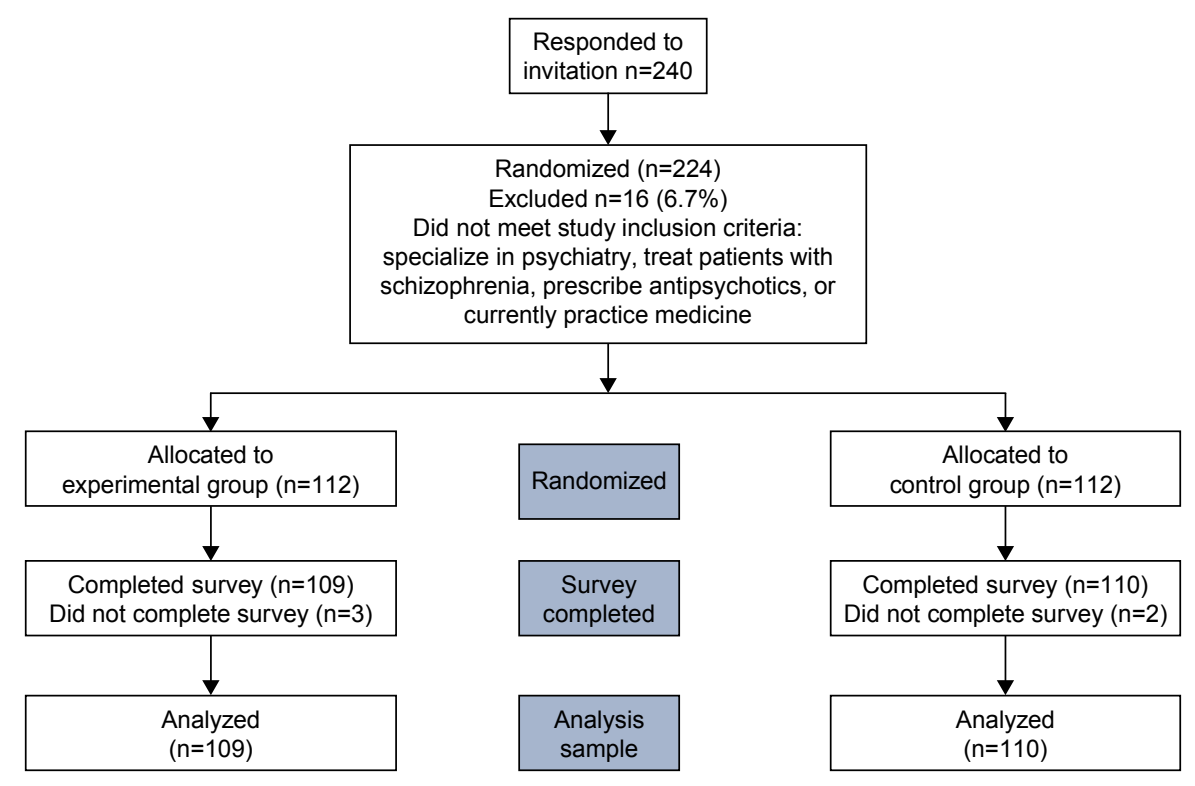

Figure 2 CONSORT diagram. 
Table I Respondent demographic and practice characteristics

\begin{tabular}{|c|c|c|c|c|c|}
\hline \multirow[t]{2}{*}{ Characteristic } & \multicolumn{2}{|c|}{ Experimental $^{a}(n=109)$} & \multicolumn{2}{|c|}{ Control $^{b}(n=I \mid 0)$} & \multirow{2}{*}{$\begin{array}{l}\chi^{2} \\
\text {-value }\end{array}$} \\
\hline & Frequency & $\%$ & Frequency & $\%$ & \\
\hline \multicolumn{5}{|l|}{ Age (years) } & \multirow[t]{7}{*}{0.457} \\
\hline $20-29$ & 2 & 1.8 & 2 & 1.8 & \\
\hline $30-39$ & 53 & 48.6 & 54 & 49.1 & \\
\hline $40-49$ & 27 & 24.8 & 21 & 19.1 & \\
\hline $50-59$ & 12 & 11.0 & 21 & 19.1 & \\
\hline $60-69$ & 12 & 11.0 & 7 & 6.4 & \\
\hline $70-79$ & 3 & 2.8 & 4 & 3.6 & \\
\hline \multicolumn{5}{|l|}{ Sex } & \multirow[t]{2}{*}{0.545} \\
\hline Female & 61 & 56.0 & 66 & 60.0 & \\
\hline \multicolumn{5}{|l|}{ Race/ethnicity } & \multirow[t]{4}{*}{0.772} \\
\hline White & 68 & 62.4 & 65 & 59.1 & \\
\hline Non-white & 37 & 33.9 & 41 & 37.3 & \\
\hline Prefer not to answer & 4 & 3.7 & 4 & 3.6 & \\
\hline \multicolumn{5}{|l|}{ Degree } & \multirow[t]{5}{*}{0.407} \\
\hline Medical doctor & 83 & 76.1 & 93 & 84.5 & \\
\hline Nurse practitioner & 14 & 12.8 & 9 & 8.2 & \\
\hline Physician's assistant & 3 & 2.8 & I & 0.9 & \\
\hline Doctor of osteopathic medicine & 9 & 8.3 & 7 & 6.4 & \\
\hline \multicolumn{5}{|l|}{ Practice experience (years) } & \multirow[t]{5}{*}{0.906} \\
\hline$<1$ & 8 & 7.4 & 8 & 7.3 & \\
\hline $1-5$ & 32 & 29.4 & 34 & 30.9 & \\
\hline $6-10$ & 26 & 23.9 & 22 & 20.0 & \\
\hline$>10$ & 42 & 38.5 & 46 & 41.8 & \\
\hline \multicolumn{5}{|c|}{ Number of patients with schizophrenia provider is currently treating } & \multirow[t]{6}{*}{0.829} \\
\hline $1-4$ & 11 & 10.1 & 7 & 6.4 & \\
\hline $5-10$ & 16 & 14.7 & 15 & 13.6 & \\
\hline $\mathrm{II}-20$ & 20 & 18.3 & 25 & 22.7 & \\
\hline $21-50$ & 28 & 25.7 & 28 & 25.5 & \\
\hline$>50$ & 34 & 31.2 & 35 & 31.8 & \\
\hline \multicolumn{6}{|l|}{ How provider determines adherence } \\
\hline Ask an informant & 103 & 94.5 & 102 & 92.7 & 0.593 \\
\hline Ask the patient explicitly & 98 & 89.9 & 105 & 95.5 & 0.115 \\
\hline Patient symptomatology & 91 & 83.5 & 84 & 76.4 & 0.188 \\
\hline Drug plasma level & 44 & 40.4 & 48 & 43.6 & 0.624 \\
\hline Contact pharmacy & 49 & 45.0 & 43 & 39.1 & 0.379 \\
\hline Pill counting & 32 & 29.4 & 33 & 30.0 & 0.917 \\
\hline Adherence assessment scale & 7 & 6.4 & 8 & 7.3 & 0.803 \\
\hline Log book & 5 & 4.6 & 6 & 5.5 & 0.769 \\
\hline Other & 7 & 6.4 & 3 & 2.7 & 0.637 \\
\hline
\end{tabular}

Notes: ${ }^{W}$ With patient drug adherence information. ${ }^{b}$ Without patient drug adherence information. ${ }^{c} P$-values are from Pearson chi-square test of independence.

condition, participants in both the experimental and control groups received 2 identical validation case vignettes. In the first validation case, both groups did not receive any adherence information, whereas, in the second validation case, both groups received credible PDAI. A Wald $\chi^{2}$ test revealed no statistically significant differences in treatment choices between the 2 groups $(P=0.553$ in the vignette where the patient was nonadherent, whereas $P=0.071$ in the vignette where the patient was adherent).

\section{Nonadherent cases}

Three vignettes presented nonadherent patients, 2 with a severe disease and 1 with a mild disease. A statistically significant increase in the preference for using LAIs by prescribers with access to credible PDAI (77.7\% vs $25.8 \%$ control; $P<0.001)$ was observed for these patients (Figure 3A). This finding was large in magnitude and was consistent across patients with either mild (case 7, 62.4\% vs $9.1 \%[P<0.001]$ ) or severe disease (case $4,82.6 \%$ vs $5.5 \%$ $[P<0.001]$; case $6,88.1 \%$ vs $62.7 \%[P<0.001])$.

\section{Adherent, poorly controlled cases}

Two vignettes described adherent but poorly controlled patients. For an adherent patient who had recently been experiencing paranoid delusions, a Wald $\chi^{2}$ test indicated that overall differences in treatment patterns were not 

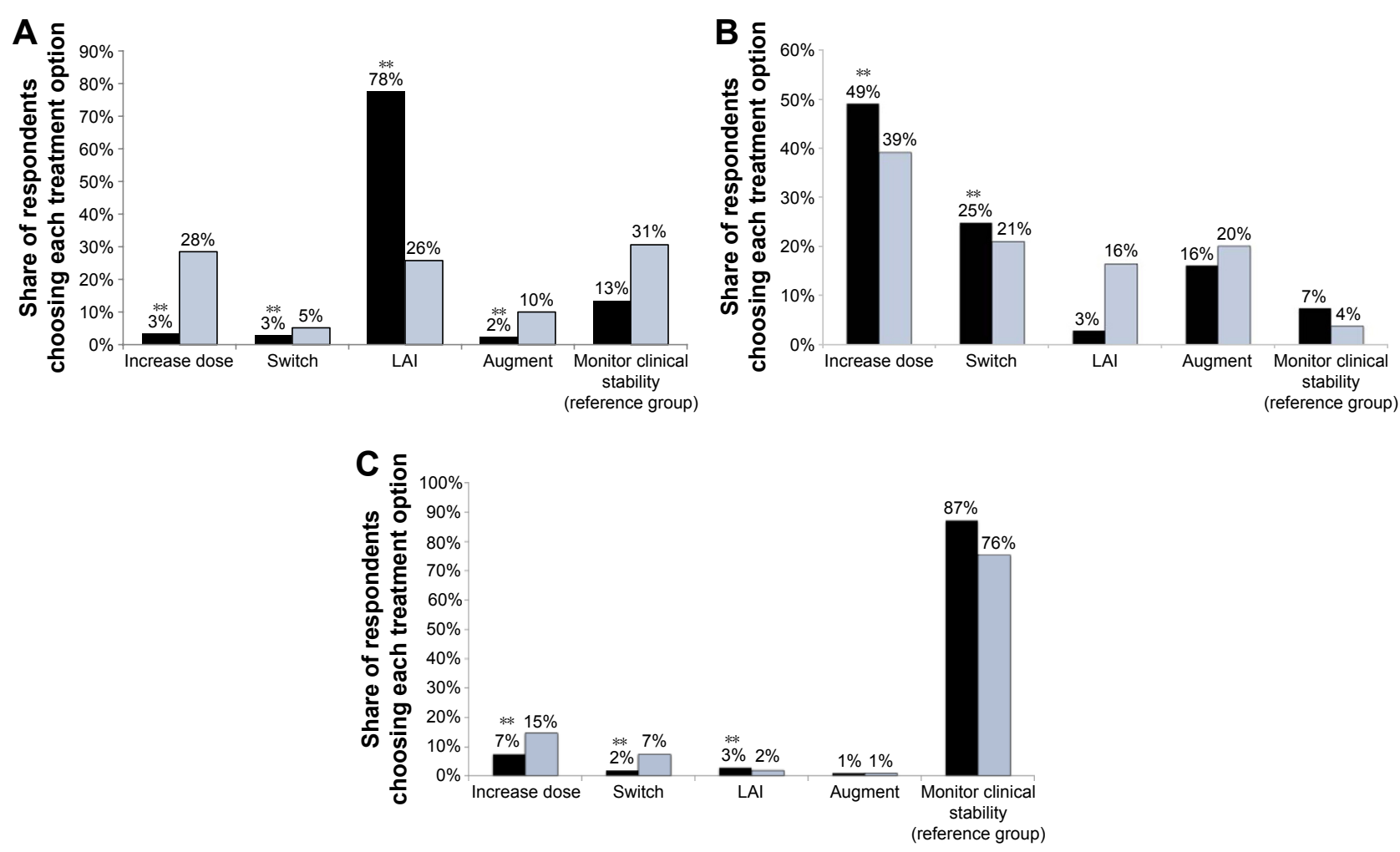

- PDAI $\square$ No PDAI

Figure 3 Effect of PDAI on treatment decisions for (A) nonadherent patients (results represent average from 3 vignettes), (B) adherent but poorly controlled patients (results represent average from 2 vignettes), and (C) adherent and well-controlled patients. The $P$-values were calculated based on a multinomial logistic regression performing pairwise comparison of each treatment decision pair across the 2 groups of respondents, adjusting for multiple comparisons using false discovery rate-adjustment.

Notes: $* * P<0.01$. The bars without an asterisks are not statistically significant.

Abbreviations: PDAI, patient drug adherence information; LAI, long-acting injectable.

significantly different $(P=0.054)$ between providers who had access to PDAI and those who did not. Providers in both groups were less likely to prescribe LAIs (2.8\% experimental and $3.6 \%$ control; $P=0.037$ ) and were more likely to recommend increasing the dose of antipsychotic medication $(65.1 \%$ experimental and $60.9 \%$ control; $P<0.001)$. Providers with access to credible PDAI were more likely to monitor clinical stability ( $11.9 \%$ experimental vs $2.7 \%$ control).

For a case representing an adherent patient recently hospitalized for a psychotic episode, significantly fewer providers with credible PDAI prescribed LAIs (2.8\% experimental vs $29.1 \%$ control; $P<0.001$ ), and a significantly higher proportion recommended increasing the dose of antipsychotic medication (33.0\% experimental vs $17.3 \%$ control; $P<0.001)$.

Across the 2 cases, providers with access to credible PDAI were more likely to increase the dose $(49.1 \%$ vs $39.1 \%$ control; $P<0.001)$ and switch medications ( $24.8 \%$ vs $20.9 \%$ control; $P<0.001$ ), but were less likely to switch to an LAI ( $2.8 \%$ vs $16.4 \%$ for control; $P=0.061)$. Figure $3 \mathrm{~B}$ summarizes the prescribers' treatment approaches in response to these 2 vignettes.
Adherent, well-controlled case

The survey included 1 vignette representing an adherent, wellcontrolled patient. Prescribers in both experimental and control groups were most likely to recommend monitoring clinical stability ( $87.2 \%$ experimental vs $75.5 \%$ control; $P=\mathrm{NS})$. A $\chi^{2}$ test indicated that the differences across all choices by group were not statistically significant $(P=0.099$; Figure 3C).

\section{Non-pharmacologic interventions}

The groups did not differ on choice of non-pharmacologic intervention recommendations for the 2 validation cases (case 1, $P=0.621$; case $8, P=0.780$ ). For the 3 nonadherent cases, respondents with access to credible PDAI were more likely to prescribe adherence-related motivational interviewing in 2 of the 3 cases (case $4,15.6 \%$ experimental vs $1.8 \%$ control $[P<0.001]$; case $6,22.9 \%$ vs $24.6 \%[P=0.780]$; case $7,54.1 \%$ vs $40.0 \%$ [ $P=0.036])$. For the 2 adherent but poorly controlled patients, respondents with access to credible PDAI were less likely to recommend adherence-related intervention in both cases, although their responses were significantly different relative to those from respondents 
without adherence information in only 1 of the 2 cases (case 3, $P=0.643$; case 5, $P<0.001$ ). For the adherent and well-controlled patient, all respondents were more likely to recommend supportive employment or a volunteer job, although these results were stronger for providers with credible PDAI (48.6\% experimental vs $31.8 \%$ control; $P<0.001$ ). More respondents without credible PDAI preferred intensive case management for this patient $(15.5 \%$ vs $3.7 \%$ in the experimental group; reference group).

\section{Discussion}

This study used a randomized clinical vignettes survey design to illustrate the effect of credible PDAI on prescribers' recommendations for modifying the current antipsychotic medication treatment regimen of patients with schizophrenia. The results underscore the value of credible and objective medication adherence information in making informed clinical decisions for managing patients with schizophrenia.

For nonadherent patients, prescribers' access to credible PDAI significantly increased the likelihood of recommending adherence-remediation interventions such as the prescription of LAI medications and adherence-related motivational interviewing. The study also showed that for nonadherent patients, the providers without access to credible PDAI were inclined to select treatment options that would be unlikely to maximize health benefits, such as increasing the dose or augmentation with a second antipsychotic. For adherent, poorly controlled patients who were not presenting with active psychosis or hospitalized for psychotic behavior, respondents with access to credible PDAI were less likely to prescribe an LAI and more likely to change the dose. Thus, access to credible PDAI may decrease unwarranted prescribing decisions. LAIs are generally recommended for patients with suspected nonadherence because they simplify dosing regimens and have the potential to improve adherence. ${ }^{19}$ Psychoeducation and motivational interviewing are the non-pharmacologic interventions frequently recommended to promote adherence. ${ }^{1}$

By contrast, for adherent patients experiencing significant psychoses (ie, hospitalized), the treatment recommendations differed significantly between groups, that is, those with PDAI were less likely to prescribe, perhaps unnecessary, LAI formulations of antipsychotics. This finding likely reflects the persistent challenges in managing treatment-refractory disease, where adherence information might be more critical for more severe symptom expression compared to poorly controlled but not actively psychotic patients. In both cases, the availability of PDAI has the potential to contribute to decisions to modify treatment plans, if for no other reason, to differentiate between persistent and intractable disease and noncompliance with medication. The most frequent treatment choice for poorly controlled patients in both experimental and control groups was increasing the medication dose, indicating that a large number of respondents in the control group assumed the patients to be adherent. Previous studies have recommended therapeutic monitoring by examining plasma levels of antipsychotics in the case of poor response before making treatment changes, ${ }^{20,21}$ although this method has limitations such as interindividual variability and unavailability of routine plasma assay. ${ }^{8,22}$

In the real-world, subjective assessments of adherence have been the most commonly used measures to estimate adherence, although validity of these methods is questionable. ${ }^{23}$ For example, physician-reported estimates of patient adherence to oral antipsychotics had a false-positive rate of $94 \%,{ }^{11}$ suggesting a clear discordance between physician perceptions and actual adherence and highlighting the need for credible adherence estimates. Furthermore, a frequently endorsed method used to assess medication adherence is assessment of patient symptoms, which is confounded by medication effectiveness. Perceptions of adherence and subsequent prescribing behavior are also shaped by characteristics and actions of both patient (eg, compliance, socioeconomic status) and provider (gender, age, practice setting). ${ }^{24}$

Objective measures of adherence, such as pharmacy claims or electronic monitoring, are considered to be more credible $^{25}$ than patient self-reports or provider estimations. It should be noted that, although objective, these are proxy methods of measuring adherence. ${ }^{8}$ The MEMSCap, for instance, registers opening of the bottle cap, but not medication ingestion.

A digital medicine system that is being developed for patients with serious mental illness uses medication with an embedded sensor that detects an ingestion event. ${ }^{13,14}$ The usability of this system has been demonstrated in patients with schizophrenia. ${ }^{26}$ Availability of credible PDAI via this system may provide an opportunity for early and targeted intervention. Until credible PDAI becomes widely available, strategies that focus on prescriber investigation of adherence at the point of care - through dialogue and collaboration with patients and their caregivers - are needed in order to identify the most appropriate therapy. Our findings underscore the importance of having available a long-acting therapy option that can help to mitigate challenges in ascertaining true adherence to treatment in the absence of more reliable methods in the clinical setting. 
Relapse is one of the most frequently cited drivers of costs associated with schizophrenia. ${ }^{27}$ Because nonadherence is a critical factor that leads to higher rates of relapse, credible approaches to monitoring adherence may decrease medical costs. A separate analysis of results from this survey found that access to credible PDAI would lead to a decrease in annual medical cost of $\$ 3560$ for nonadherent patients with schizophrenia. ${ }^{28}$ Thus, access to accurate adherence information has important economic implication in addition to clinical benefits.

This study has several strengths. It is the first study to incorporate clinical case vignettes to quantify preferences of psychiatric health care providers for treating patients with schizophrenia based on the availability of adherence information. The eligibility criteria ensured that the recruited providers had the appropriate backgrounds to participate in the survey, currently practiced medicine, prescribed antipsychotics, and treated patients with schizophrenia. Random assignment of providers to either the experimental or control group removed or reduced potential bias.

\section{Limitations}

The vignettes were limited to 8 clinical cases and represent only a fraction of the heterogeneous population of patients with schizophrenia seen in clinical practice. Furthermore, the decision process for a hypothetical case might not entirely reflect real-world treatment decisions. Providers were asked to make treatment decisions without considering restrictions imposed by insurance plans, limited resources, and other prescribing constraints such as formulary restrictions or requirements for prior authorization. Additionally, providers were asked to make decisions in the absence of additional clinical information that could be obtained during patientphysician dialogue in usual clinical practice. Therefore, providers' responses captured in the survey may be more synthetic than would be expected upon initial review of the vignettes. Finally, the study utilized a convenience sample; therefore, it was not designed to generate nationally representative conclusions. Potential study participants were identified from commercially available list of psychiatric prescribers of antipsychotic medication for schizophrenia that provided only contact information. As a convenience sample, we make no claim of the generalizability of study results. However, because we randomly assigned participants into 2 groups that were not statistically different across the characteristics measured in this study, we infer that the differences on treatment choice between groups are the result of credible compliance information presented within the experimental group's vignettes.

\section{Conclusion}

In this study, provider access to credible PDAI led to significantly increased use of adherence-remediating interventions for nonadherent patients with schizophrenia and decreased use of adherence-targeted interventions for adherent patients. Access to adherence information is particularly important for treating real-world patients in typical clinical settings where blood-level assessments of antipsychotic treatments are rarely available and where LAI preparations are underused. Access to credible PDAI has the potential to improve outcomes and reduce health care costs associated with schizophrenia, and will also help clinicians evaluate potential side effects more accurately if they know whether the relationship with medication is plausible or not.

\section{Acknowledgments}

Funding for this study was provided by Otsuka Pharmaceutical Development \& Commercialization, Inc. Editorial support for the development of this manuscript was provided by Vandana Sharma, $\mathrm{PhD}$, at $\mathrm{C} 4$ MedSolutions, LLC (Yardley, PA), a CHC Group company, and was funded by Otsuka Pharmaceutical Development \& Commercialization, Inc. This study was presented at the American Society of Clinical Psychopharmacology Annual Meeting, held on May 30-June 3, 2016, in Scottsdale, AZ, and the 29th Annual U.S. Psychiatric and Mental Health Congress, held on October 21-24, 2016, in San Antonio, TX.

\section{Disclosure}

Jason Shafrin, Suepattra G May, Anshu Shrestha, and Darius Lakdawalla are employees of Precision Health Economics, a health care consulting firm that received funding for this study from Otsuka. Charles Ruetsch and Nicole Gerlanc are employees of Health Analytics, LLC, a contract research company compensated by Otsuka to conduct the study. Felicia Forma is an employee of Otsuka Pharmaceutical Development \& Commercialization, Inc. Ainslie Hatch is an employee of ODH, Inc and was an employee of Otsuka Pharmaceutical Development and Commercialization during this research project. Jean-Pierre Lindenmayer is a paid consultant for Otsuka and has received research support from Janssen, Alkermes, Forum, Neurocrine, and Avanir. The authors report no other conflicts of interest in this work.

\section{References}

1. Haddad PM, Brain C, Scott J. Nonadherence with antipsychotic medication in schizophrenia: challenges and management strategies. Patient Relat Outcome Meas. 2014;5:43-62.

2. Novick D, Haro JM, Suarez D, Perez V, Dittmann RW, Haddad PM. Predictors and clinical consequences of non-adherence with antipsychotic medication in the outpatient treatment of schizophrenia. Psychiatry Res. 2010;176(2-3):109-113. 
3. Ascher-Svanum H, Faries DE, Zhu B, Ernst FR, Swartz MS, Swanson JW. Medication adherence and long-term functional outcomes in the treatment of schizophrenia in usual care. J Clin Psychiatry. 2006; 67(3):453-460.

4. Lindenmayer JP, Liu-Seifert H, Kulkarni PM, et al. Medication nonadherence and treatment outcome in patients with schizophrenia or schizoaffective disorder with suboptimal prior response. J Clin Psychiatry. 2009;70(7):990-996.

5. Subotnik KL, Nuechterlein KH, Ventura J, et al. Risperidone nonadherence and return of positive symptoms in the early course of schizophrenia. Am J Psychiatry. 2011;168(3):286-292.

6. Bayle FJ, Tessier A, Bouju S, Misdrahi D. Medication adherence in patients with psychotic disorders: an observational survey involving patients before they switch to long-acting injectable risperidone. Patient Prefer Adherence. 2015;9:1333-1341.

7. Offord S, Lin J, Mirski D, Wong B. Impact of early nonadherence to oral antipsychotics on clinical and economic outcomes among patients with schizophrenia. Adv Ther. 2013;30(3):286-297.

8. Sajatovic M, Velligan DI, Weiden PJ, Valenstein MA, Ogedegbe G. Measurement of psychiatric treatment adherence. J Psychosom Res. 2010;69(6):591-599.

9. Velligan DI, Wang M, Diamond P, et al. Relationships among subjective and objective measures of adherence to oral antipsychotic medications. Psychiatr Serv. 2007;58(9):1187-1192.

10. Byerly MJ, Thompson A, Carmody T, et al. Validity of electronically monitored medication adherence and conventional adherence measures in schizophrenia. Psychiatr Serv. 2007;58(6):844-847.

11. Stephenson JJ, Tunceli O, Gu T, et al. Adherence to oral secondgeneration antipsychotic medications in patients with schizophrenia and bipolar disorder: physicians' perceptions of adherence vs. pharmacy claims. Int J Clin Pract. 2012;66(6):565-573.

12. Garfein RS, Collins K, Munoz F, et al. Feasibility of tuberculosis treatment monitoring by video directly observed therapy: a binational pilot study. Int J Tuberc Lung Dis. 2015;19(9):1057-1064.

13. Profit D, Rohatagi S, Zhao C, Hatch A, Docherty JP, Peters-Strickland TS. Developing a digital medicine system in psychiatry: ingestion detection rate and latency period. J Clin Psychiatry. 2016;77(9): e1095-e1100.

14. Rohatagi S, Profit D, Hatch A, Zhao C, Docherty JP, Peters-Strickland TS. Optimization of a digital medicine system in psychiatry. J Clin Psychiatry. 2016;77(9):e1101-e1107.

15. Kulkarni J, Reeve-Parker K. Psychiatrists' awareness of partial- and non-adherence to antipsychotic medication in schizophrenia: results from the Australian ADHES survey. Australas Psychiatry. 2015; 23(3):258-264.
16. Geerts P, Martinez G, Schreiner A. Attitudes towards the administration of long-acting antipsychotics: a survey of physicians and nurses. $B M C$ Psychiatry. 2013;13:58.

17. Tang Y, Chang CC, Lave JR, Gellad WF, Huskamp HA, Donohue JM. Patient, physician and organizational influences on variation in antipsychotic prescribing behavior. J Ment Health Policy Econ. 2016;19(1): 45-59.

18. Mark TL, Kassed CA, Vandivort-Warren R, Levit KR, Kranzler HR. Alcohol and opioid dependence medications: prescription trends, overall and by physician specialty. Drug Alcohol Depend. 2009;99(1-3): 345-349.

19. Brissos S, Veguilla MR, Taylor D, Balanza-Martinez V. The role of long-acting injectable antipsychotics in schizophrenia: a critical appraisal. Ther Adv Psychopharmacol. 2014;4(5):198-219.

20. Lopez LV, Kane JM. Plasma levels of second-generation antipsychotics and clinical response in acute psychosis: a review of the literature. Schizophr Res. 2013;147(2-3):368-374.

21. Lopez LV, Kane JM. Recommendations for the monitoring of serum concentrations of antipsychotic drugs in the treatment of schizophrenia. J Clin Psychiatry. 2015;76(9):1249-1250.

22. Grundmann M, Kacirova I, Urinovska R. Therapeutic drug monitoring of atypical antipsychotic drugs. Acta Pharm. 2014;64(4):387-401.

23. Velligan DI, Lam YW, Glahn DC, et al. Defining and assessing adherence to oral antipsychotics: a review of the literature. Schizophr Bull. 2006;32(4):724-742.

24. Franz M, Ranger J, Hanewald B, Gallhofer B, Lay B. Influences on therapist's decisions for neuroleptic treatment in schizophrenia: the role of characteristics of the patient and the physician. Pharmacopsychiatry. 2012;45(7):261-268.

25. Velligan DI, Weiden PJ, Sajatovic M, et al. The expert consensus guideline series: adherence problems in patients with serious and persistent mental illness. J Clin Psychiatry. 2009;70 Suppl 4:1-46; quiz 47-48.

26. Peters-Strickland T, Pestreich L, Hatch A, et al. Usability of a novel digital medicine system in adults with schizophrenia treated with sensor-embedded tablets of aripiprazole. Neuropsychiatr Dis Treat. 2016;12:2587-2594

27. Lafeuille MH, Gravel J, Lefebvre P, et al. Patterns of relapse and associated cost burden in schizophrenia patients receiving atypical antipsychotics. J Med Econ. 2013;16(11):1290-1299.

28. Shafrin J, Schwartz TT, Lakdawalla DN, Forma FM. Estimating the value of new technologies that provide more accurate drug adherence information to providers for their patients with schizophrenia. J Manag Care Spec Pharm. 2016;22(11):1285-1291. 


\section{Supplementary materials}

Table SI Distribution of key patient characteristics in vignettes

\begin{tabular}{|c|c|c|c|c|c|c|c|c|c|}
\hline \multirow[t]{2}{*}{ Factors } & \multirow[t]{2}{*}{ Group } & \multicolumn{8}{|c|}{ Patient vignette } \\
\hline & & 1 & 2 & 3 & 4 & 5 & 6 & 7 & 8 \\
\hline Adherence & Experimental & No & Yes & Yes & Yes & Yes & Yes & Yes & Yes \\
\hline information available & Control & No & No & No & No & No & No & No & Yes \\
\hline Patient is adherent & Both groups & No & Yes & Yes & No & Yes & No & No & Yes \\
\hline Age (years) & & $<40$ & $<40$ & $<40$ & $<40$ & $\geq 40$ & $\geq 40$ & $\geq 40$ & $\geq 40$ \\
\hline Sex & & Male & Male & Female & Female & Male & Male & Female & Female \\
\hline Severity & & Mild & Severe & Mild & Severe & Mild & Severe & Mild & Severe \\
\hline
\end{tabular}

Notes: Vignettes I and 8 represent the validation test where both arms received the same adherence information. Vignette 2 is an adherent, well controlled case. Vignettes 3 and 5 are adherent, poorly controlled cases. Vignettes 4, 6 and 7 represent non-adherent patients.

Table S2 Descriptions of clinical vignettes

\begin{tabular}{lll}
\hline $\begin{array}{l}\text { Case } \\
\text { name }\end{array}$ & $\begin{array}{l}\text { Case } \\
\text { number }\end{array}$ & Case description \\
\hline Toby & I & Toby is a 28-year-old male with a 5-year history of schizophrenia. Toby began to have social withdrawal and isolation
\end{tabular}

Toby I Toby is a 28-year-old male with a 5-year history of schizophrenia. Toby began to have social withdrawal and isolation around age 20 when he dropped out of sports, went from being an excellent student to barely passing his classes, and began spending much of his free time alone watching YouTube videos. He was using alcohol, marijuana, and methamphetamines. He had progressively paranoid delusions to the point where his mother had him admitted to an inpatient psychiatric hospital at age 23. Toby's first psychiatric admission lasted 4 days. He was diagnosed with paranoid schizophrenia and enrolled into an investigational study that prescribed risperidone (Risperdal) $(4 \mathrm{mg} / \mathrm{d})$. While he was taking risperidone, his hallucinations decreased somewhat but did not disappear. He had a good relationship with his treating outpatient physicians, who noted mild, persistent negative symptoms. Toby was readmitted to a psychiatric hospital for an episode of acute paranoid schizophrenia. He has been out of the hospital for one week and reports that he has not used drugs or alcohol since his hospitalization.

Jean 7 Jean is a 43 -year-old female who has been taking clozapine (Clozaril) $(300 \mathrm{mg} / \mathrm{d})$ for 6 months. Jean was diagnosed with schizophrenia 15 years ago, but she's never agreed with the diagnosis. She prefers to think of herself as someone who can hear voices and that this is a gift. The voices are only a problem when they become abusive and tell her what an 'evil, spiteful witch' she is. Prior to starting clozapine, she had tried 'loads of different pills' (including: olanzapine, quetiapine, and lurasidone), none of which had been effective at making the 'voices nice again'. Jean does not know whether she wants to stick with the clozapine. She wonders if she might just be better off without it. Based on data from electronic pill cap monitors, Jean does not take her clozapine every day. Today's blood plasma levels confirm this finding.

Robert 6 Robert is a 44-year-old male with a 17-year history of schizophrenia who has had multiple psychiatric admissions usually in the context of non-adherence. Admissions have been characterized by gradually increasing isolation, preoccupations, and insomnia. These symptoms escalate eventually to agitation, intense paranoid ideation with at times yelling at strangers and total neglect of his ADLs. Robert has been living in a group home with 8 other residents where he is responsible for taking his own medication. He usually does attend his monthly outpatient visits and also attends a club house. His medications were lurasidone (Latuda) $(80 \mathrm{mg} / \mathrm{d})$ and metformin $[1,000 \mathrm{mg} / \mathrm{d}]$. Over the past few weeks Robert has become increasingly unpredictable, responding to internal stimuli with bursts of uncontrollable laughing and swearing. He also believes the other group home members are out to get him. His psychiatrist called 911 to have Robert admitted to the inpatient services. After a 6 day intensive inpatient stay Robert re-stabilized on lurasidone (Latuda) (I20 mg/d) and was discharged back to the group home. Two weeks after discharge, his psychiatrist reviewed Robert's electronic cap monitor data and blood plasma levels and found that Robert had only taken half of the pills he was supposed to take and had low plasma levels.

Kay 4 Kay is a 34-year-old female with an 8-year history of schizo-affective disorder, with multiple depressive episodes that led to drug overdoses and three suicide attempts. She has received four courses of electroconvulsive therapy, the first of which she had at age 28 , and has been prescribed multiple psychotropic medications, including antipsychotics and antidepressants at various time points. Her medications have included paroxetine, risperidone, lurasidone, and aripiprazole. She had been living with her grandmother until recently. After her grandmother's death, she moved into a group home for patients with psychiatric illnesses. While she smokes two packs of cigarettes daily, she reports no alcohol consumption or use of illicit drugs. She is currently receiving a combined regimen of clozapine (Clozaril) [450 mg/d] and lithium (Eskalith) ( $900 \mathrm{mg} / \mathrm{d}$ ), however, she is not doing any better than she has on previous medications. The group home supervisor reports Kay is showing signs of active psychosis including isolation and apparent responses to internal stimuli. Data from electronic pill cap monitors indicate she often goes multiple days without taking some or all of her medications and Kay's clozapine blood plasma levels are a little low. 
Table S2 (Continued)

Case Case Case description

name number

Samantha 3 Samantha is a 28-year-old female with a 5-year history of schizophrenia. Samantha began to have social withdrawal

(Sam) and isolation around age 19 after completion of HS. However, she did attend the first year of an out-of-town college. She made a bad adjustment to the school. She went from being an excellent student to gradually having difficulties in her courses, staying away from classes and having difficulties sleeping. She was seen by the college campus psychiatrist who recommended return to her home town and attendance at a community college. However, she had progressively paranoid delusions to the point where her mother had her brought to a psychiatric emergency room. After being placed on risperidone (Risperdal) $(4 \mathrm{mg} / \mathrm{d})$ at the time of diagnosis, her paranoid ideation decreased somewhat, but did not disappear. Based on a recent electronic cap monitor data and blood plasma levels, her medication adherence has been consistent while she attends her outpatient visits and her paranoid delusions are under partial control.

Aaron 5 Aaron is a 40-year-old male with a 16-year history of schizophrenia and was admitted to the hospital three weeks ago for observation by an ER physician who had expressed concern about Aaron's behavior. Aaron has been living in a group home with 8 other residents and was responsible for taking his own medication. He has had intermittent employment in lawn care around the city and seemed content with the work. Over the past few weeks Aaron had become increasingly unpredictable, responding to internal stimuli as well as cursing, tickling himself and laughing uncontrollably. A recent manic episode resulted in the visit to the ER. Aaron also believed that the other group home members were out to get him and that others questioned his sexual orientation. Based on electronic pill count monitors and his most recent blood plasma levels, Aaron takes his aripiprazole (Abilify) $(20 \mathrm{mg} / \mathrm{d})$ and risperidone (Risperdal) (8 mg/d) regularly and rarely misses a dose.

Adam 2 Adam is a 3l-year-old male diagnosed with schizophrenia 7 years ago. During college, Adam began to hear voices that told him he was no good. Employed at a local supermarket, Adam began to believe that his boss was planting small video cameras to catch him making mistakes. Adam became increasingly agitated at work, particularly during busy times, and began "talking strangely" to customers. He lost his job at the local supermarket, and relationships with family members broke down as he feared that they would betray him to spies. He became increasingly confused and agitated. His parents took him to the hospital where he was admitted and administered haloperidol (Haldol) ( 5 mg, injected) by his psychiatrist. The medication caused painful twisting and contractions of his muscles. He was switched to olanzapine (Zyprexa) $(10 \mathrm{mg} / \mathrm{d})$ and has had fewer side effects. Based on data from electronic pill cap monitors, Adam seldom misses a dose of his olanzapine, and his blood plasma levels are as expected for his dose.

Margaret $8 \quad$ Margaret is a 60-year-old female with a 27-year history of undifferentiated schizophrenia and has received multiple therapies over that time. In the most recent episode, last month, in which she was hospitalized, she presented with auditory hallucinations, disorganized speech, inappropriate affect with an occasionally euphoric mood, and grossly disorganized behavior and excitability. Impaired glucose tolerance due to adiposity and metabolic syndrome (weight 226 lbs., 5' I, BMI: 42.7) was observed and was treated with metformin (500 mg/d). While tapering off quetiapine, her doctor prescribed lurasidone (Latuda) $(80 \mathrm{mg} / \mathrm{d})$ and titrated up to a daily dosage of $120 \mathrm{mg}$ within 30 days. No substantial improvement was achieved with this regimen within 30 days. Data from the electronic pill cap monitors and plasma level indicators support that Margaret is adherent to her medication.

Note: Adherence information is given in italics.

Abbreviations: ADLs, activities of daily living; HS, high school; ER, emergency room; BMI, body mass index.

Patient Preference and Adherence

Dovepress

\section{Publish your work in this journal}

Patient Preference and Adherence is an international, peer-reviewed, open access journal that focuses on the growing importance of patient preference and adherence throughout the therapeutic continuum. Patient satisfaction, acceptability, quality of life, compliance, persistence and their role in developing new therapeutic modalities and compounds to optimize clinical outcomes for existing disease states are major areas of interest for the journal. This journal has been accepted for indexing on PubMed Central. The manuscript management system is completely online and includes a very quick and fair peer-review system, which is all easy to use. Visit http://www. dovepress.com/testimonials.php to read real quotes from published authors. 